Jacqueliu du Val (camille) GLANURES ENTOMOLOGIQUES 


$$
\begin{aligned}
& \text { Q } 463 \\
& \text { D98 } \\
& \text { r.1 } \\
& \text { Ent. }
\end{aligned}
$$

e80076 
Voué en entier à l'étude de l'entomologie, et poursuivant toujours mon grand travail sur les Coléoptères d'Europe, j’ai souvent entre les mains des matériaux précieux et je suis à même de faire de nombreuses observations. Ces glanures ont pour but de publier les travaux spéciaux et les principales remarques qui ne peuvent trouver place dans mon Genera, lequel ne prend déjà que trop d'extension. Elles paraîtront par petits cahiers à des époques indéterminées. Chaque cahier contiendra toujours l'essai monographique ou le synopsis des espèces européennes d'un ou deux genres, des descriptions d'espèces nouvelles -remarquables, des notes critiques, des discussions diverses, et enfin des synonymies plus ou moins nombreuses. Cinq cahiers paginés d'une manière consécutive forme- 
ront un volume dont je donnerai alors la table. Leur tirage n’aura lieu qu'à deux cents exemplaires.

Puissent les petits travaux etles observations contenues dans ces glanures être utiles à la science et recevoir un bon accueil.

$$
\text { JACQ, DU V. }
$$

Paris, 10 octobre 1859. 


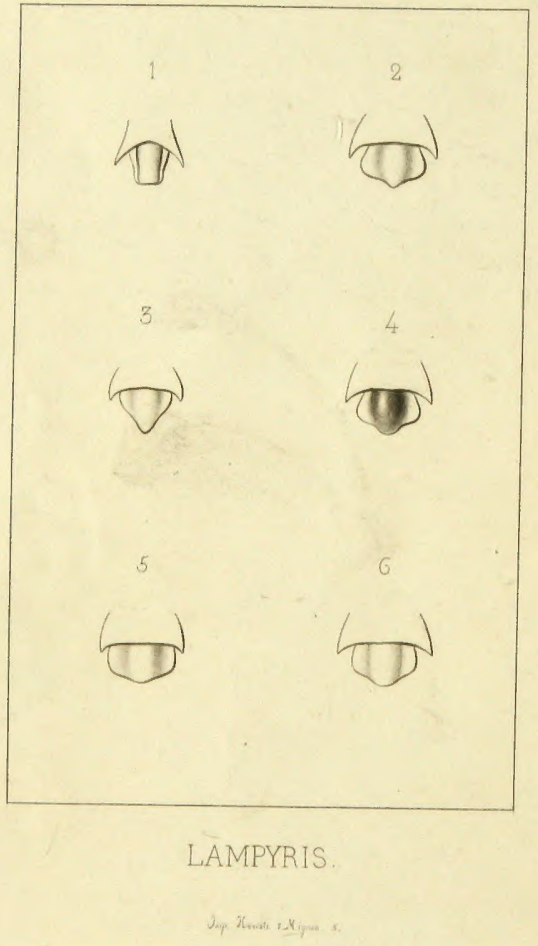




\section{GLANURES ENTOMOLOGIQUES}

ov

RECUEIL DE NOTES MONOGRAPHQUUS, DESCRIPTIONS, CRITIQUES, REMARQUES ET STNONYMIES DIVERSES,

Par as. Jaceurcus be val (Camille).

CAHIER 1. PARIS, 25 OCTUBRE 1859.

\section{SY NOPS I S}

DES

\section{ESPÈCES GUROPÉnNNES DU GENRE LAIIPYRIS}

Ayant eu besoin d'étudier de près les Lampyris pour mon Genera, j'ai dû à l'obligeance de M. Reiche la communication de sa riche collection, communication d'autant plus précieuse que mon savant collègue, se proposant de faire un travail sur ces insectes, avait réuni un grand nombre de matériaux. Renonçant généreusement aux études et aux recherches qu'il avait déjà faites sur ce sujet, M. Reiche m'a engagé à publier le fruit de mes observations, et c'est ce que je fais ici, sans avoir toutefois la prétention d'avoir fait un véritable travail monographique. J'ai dû en outre à l'obligeance de MM. de Bonvouloir, Cherrolat et Delarouzée la communication de leurs richesses. 
Le genre Lampyris, tel qu'il est admis aujourd'hui, doit se diviser en deux coupes génériques bien distinctes, les Lampyris et les Lamprohiza. Je renvoie à mon Genera pour l'exposition détaillée des caractères de ces genres, et je vais me borner ici à donner de très courtes diagnoses différentielles.

\section{Gente Lampyris Geof.}

Mandibules petites, point saillantes, médiocrement étroites, droites, subparallèles ; terminées au sommet en dedans par une toute petite pointe aiguë, ciliées en outre sur leur partie dorsale. Pronotum offrant fréquemment en avant deux petites taches translucides, mais en général peu tranchées, nulles ou indistinctes chez les femelles. - Mâle. Abdomen offrant inférieurement au sommet un petit arceau supplémentaire plus ou moins distinct. Femelle. Taille généralement plus grande. Corps larviforme. Élytres tout à fait nulles, ou représentées simplement par de petits moignons en forme d'écailles, sinués postérieurement et plus ou moins aigus. Abdomen de huit segments bien distincts.

I. Moignons élytraux tout à fait nuls ou soudés, plus ou moins confondusavec le mésonotum, lequel forme une grande pièce unique sans écusson distinct \&. - Dernier segment ventral apparent à peine ou indistinctement sinué de chaque côté, avec une petite ou faible échancrure médiane. $\delta^{\star}$.

A. Moignons élytraux entièrement confondus avec le mésonotum, indistincts, ne débordant point visiblement le métanotum sur les côtés. む. 
X Huitième segment ventral profondément incisé en forme de V aigu. Corps entièrement d'un testacé fauve. .

1. r. Mauritaniea. Lin. Syst. Nat. édit. 12. Ir. $645.0^{x}$. (1767).- Olivier. Ent. II. 28. pl. 1. 5 a. $\sigma^{7}$. (1790) (type). -Fabr. Ent. Syst. I. Ir. 103. ठ, (1792).

Mâle. L. 14-15. mil. D’un testacé flave; élytres d'un testacé brunâtre; parfois plus brunes, avec un large bord latéral et une fine marge suturale testacés. Pronotum en ogive courte, pas plus long ou un peu moins long que large, sensiblement rétréci en avant, avec son rebord latéral et antérieur fortement relevé. Dernier segment dorsal de l'abdomen fortement sillonné de chaque côté etlongitudinalement convexe dans son milieu, simplement et légèrement rétréci en arrière, point distinctenent dilaté sur ses côtés qui sont presque droits, large au sommet (fig. 1). Arceaux précédents notablement prolongés à leurs angles postérieurs externes en lanières aiguës très saillantes.-Femelle. 18-22 millim. En entier d'un testacé fauve. Mésonotum en général visiblement rétréci et prolongé dans son milieu en arrière, formant par suite un angle obtus plus ou moins marqué. - Cette espèce se fait remarquer en outre entre toutes les suivantes par son pronotum très subtilement pointillé. - Espagne méridionale, Algérie.

XX. Huitième segment ventral peu profondémentincisé; l'incision généralement en V très ouvert. Corps brun en majeure partie. + .

2. r. Noetiluea. Lin. Syst. Nat. édit. 12. II. 643. $\delta^{x}$. (1767). - Fourcr. Geof. Hist. des Ins. 1. 166. 0 . $q$. 
(1764). - De Géer Mém. Iv. 31. (1774). - Olivier

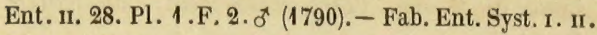
98. $\delta^{x}$. (1792). - Panz. Faun. German. 41.7. $\delta^{x}$. (17951808). - Gyllenh. Ins. Suec. 1. 318. ơ. 우 (1808) . Westw. Intr. 1. 247. $\sigma^{x}$. ㅇ . (1839). - Küst. Kaf. Eur. Ix. 25. $\sigma^{2}$. ㅇ. ( 1847) - - L. splendidula. Oliv. Ent. II. 28. Pl. 1, fig. $1 d$. ㅇ . (1790) . - Latr. Règn. anim. édit. Masson I. 219. $\sigma^{7}$. 우.

Mâle. L. 12-16 mil. D'un testacé fauve; disque du pronotum plus ou moins largement brunâtre; élytres brunes en entier; abdomen plus ou moins hrunâtre, avec le bord postérieur des segments, le rebord latéral plus largement, et les derniers arceaux inférieurs en entier, testacés. Pronotum un peu moins long que large, faiblement ou point sensiblement rétréci en avant, avec ses côtés presque droits en arrière. Dernier segment dorsal de l'abdomen légèrement convexe longitudinalement dans son milieu; notablement dilaté-arrondi sur les côtés, puis brusquement mais très brièvement rétréci au sommet (fig. 2). Arceaux précédents prolongés à leurs angles postérieurs externes en lanières aiguës en général assez saillantes. Femelle. L. 13-23 mill. D'un brun un peu soyeux; limbe du pronotum plus ou moins largement d'un jaune testacé pâle; côtés du mésonotum, et souvent aussi du métanotum, et membranes unissant entre eux les arceaux du corps, d'un rose plus ou moins vif; poitrine variée de même; abdomen inférieurement brunâtre, avec ses arceaux bordés de testacé et ses trois derniers en entier de cette couleur; pattes brunâtres, plus ou moins linées ou teintées de testacé. Mésonotum presque simplement arqué 
en arc en arrière, ou en angle tout ì fait obtus et i peine senti. - Europe.

Varicté Longipennis Motsch. Elud. Ent. 11r. 21. $0^{x}$. (1834). - La L. Longipennis Motsch. a été baséc tout sinplement sur des exemplaires à élytres un peu plus longues et un peu atténuées en arrière, avec le pronotum un peu plus court. C'est une variété insignifiante que MI. Molschulsky avait reçu des ['yrénées-Orientales, où la L. noctiluca est commune.

Variété Bellieri Reiche An. Soc. Ent. Fr. 1858.15̈̈ ơ. (ıype).- La variété bellieri comprend les exemplaires de plus grande taille, lesquels sont en général d'un brun plus foncé, avec l'abdomen plius obscur et le métapectus un peu brunâire. Ces exemplaires en outre offrent assez souvent l'écusson presque carré ou tronqué en arrière. - Le caraclère tiré de la forme de l'écusson n'offre aucune constance, le plus souvent seulement les exemplaires moyens ont cette partie postérieurement arrondie et les petits individus la présentent triangulaire. - Cette variété sc trouve dans les Pyrénées-0rientales, où j’ai découvert sa femelle, qui ne diffère de celle de la L. noctulica type que par sa taille plus grande.

Nota. J'ai vu dans la collection de M. Chevrolat un type d'Olivier qui n'est tout simplement qu'un exemplaire itroit et immature de la L. noctiluca. Un second type de la même collection se rapporte parfaitement à la vraie L. mauritanica qu’olivier a évidemment représentée pl.4, fig. $5 a$.

B. Moignons élytraux assez distincts quoique très petits et soudés, débordant trìs notablement le metanotum sur 
les côtés, mais en s'inftéchissant, sinués visiblement en arrière. $q$.

3. x. Zenkeri Germ. Reis, in Dalmat. 2.11. Pl. 10. fig. 1-3. o. + . (1817). - Küst. Kaf. Lur. 1. 16. $0^{\star}$. ㅇ. (1844). - Lampronetes cincta, Motsch. Etud. Ent. III. 20. $\delta^{x} \cdot+\cdot(185 k)$ ?

Mále. L. 12. 15 mil. D’un jaune testacé påle; pronotum avec une tache discoildale brume plus ou moins distinctement entourée d'une teinte rosée; élytres d'un brun plus ou moins clair avec une fine marge latérale et suturale testacée. Pronotum un peu ou guère moins long que large, point sensiblement ou à peine rétréci en avant, avec ses côtés presque droits en arrière. Dernier segment dorsal de l'abajomen légèrement convexe longitudinalement dans son milieu, faiblement ou à peine arrondi sur les côtés, notablement et assez longucment, mais assez graduellement rétréci en arrière, plus ou moins subacuminé au sommet (fig. 3). Arceaux précédents prolongés à leurs angles postérieurs externes en lanières assez courtes ou dents aiguës. - Femelle. L. 20 mil. D’un brun un peu soyeux; limbe du pronotum assez largement d'un fauve testacé; méso et métanotum, ainsi que la poitrine, variés de rose ou de rougeâtre. Arceaux dorsaux de l'abdomen marginés de fauve testacé. Dessous du corps et pattes d'un testacé fauve ou légèrement brunâtre, plus clair sur les bords des segments et sur les derniers en entier. Mésonotum formant un angle obtus dans son milicu en arrière. Huitième arceau ventral triangulairement incisé au sommet. - Dalmatie, Étruric.

Nota. J'ai vu dans la collection de M. Reiche un exem- 
plaire o veuant de Sicile, qui présente lo dernier arceau dorsal de l'abdomen bien plus fortement rétréci en arrière et par suite tout à fait acuminé, mais je n’ai osé le séparer de la L. Zenkeri. Du reste, la modification en question n'est qu'une exagération do celle qui existe dans la L. Zenkeri type.

II. Moignons élytraux plus ou moins développés, distincts et point soudés ni confondus avec le mésonotum, mais tout au plus à peu près égaux à la partie apparente de ce dernier ou mème plus petits. Ecusson plus ou moins distinctement séparé du mésonotum. ‥-Dernier segment ventral apparent comme dans la division I. $\sigma^{\pi}$.

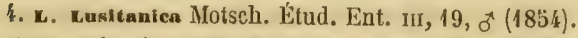
- L. mauritanica Küst. Kaf. Eur. 1х, 26. $0^{x}$, 우 (1817). Mâle. L. 13-16 mil. D’un testacé fauve mêlé d'une légère teinte rosée sur la poitrine; pronotum avec une grancle tache discoildale brune, plus ou moins distinctement entourée, surtout postéricurement, d'une teinte rosée; élytres brunes, avec une marge latérale et suturale testacée, étroite mais distincte. Pronotum à peine aussi long ou un peu moins long que large, faiblement ou à peine rétréci en avant, avec ses côtés presque droits en arric̀re. Dernier segment dorsal de l'abdomen offrant la mème forme que chez la noctiluca, mais avec la partic rétrécie apicale légèrement moins courte, un peu plus large et notalement arrondie au sommet; très-fortement convexe en outre longitudinalement dans son milieu et comme gibbeux (fig. 4). $\Lambda$ rceaux précédents prolongés à leurs anglespostérieurs externes en forme de dents aigües en général assez saillantes. - F'emellc. L. 23. D’un brun 
un peu soyeux; pronotum avec une large tache discoïdale brune entource de rose, son limbe d'un jaune testacé; métanotum varié de rose et de testacé; tous les seg. ments suivants entourés d'une teinte rose et de plus avec une étroite marge latérale et postéricure testacée; pattes d'un jaune testacé; dessous du corps de la même couleur, mais varié de rose. Écusson notablement plus large que long, légèrement arrondi en arrière, avec une toute petite et étroite échancrure méliane. Huitième segment ventral incisé en V. - Cette espèce sn fait en outre remarquer par son corps assez luisant chez le mâle, notamment sur le pronotum. - France méridionale orientale. Poriugal (Molsch.).

Nota. J'ai vu deux seuls exemplaires femelles de cette espèce, dont l'un offrait des moignons élytraux notablement plus petits que la partic apparente du mésonotum, et l'autre des moignons élytraux aussi grands que cette dernière; mais tous leurs autres caractères concordant, leurs mâles étant identiques, et leur provenance la mème, je ne puis les regarder que comme des variations de la même espèce.

b. I. Yareynii. Reiche inédit.

Mâle. L. 11-14. L’un jaune testacé; pronotum d'un testacé pâle, avec une tache discoïlale brunâtre, ou beaucoup plus claire et simplement d'un roux jaunître; ćlytres d'un brun clair, avec une fine marge latérale et suturale testacíe. Pronotum à peu près aussi long que large, nullement rétríci en avant, avee ses côtés presque droits dans leurs deux tiers postérieurs. Dernier segment dorsal de l'abdomen court, légèrement convexe longitudi- 
nalement dans son milicu, visiblement arrondi sur les côtés mais tout à fait en arrière, brusquement mais des plus Jrièvement rétréci au sommet (fig. :i). Arceaux précédents prolongés simplement à leurs angles postérieurs externes en forme de dents aiguës. - Femelle. L. 15. En entier d'un testacé fauve, avec la tête lrunître, ce qui produit par transparence une légère tache au milieu du pronotum. Écusson à peine plus large que long, arrondi en ar. rière. Huitiène segment ventral incisé en V. - Cette egpèce se fait remarquer par sa forme étroite et parallèle. C'est la plus étroite de toutes celles qui me sont connucs. - Corse, Sardaigne, Sicile.

La L. Lareynii a été rapportée de Corse par mon malhoureux ami Lareynie, ¿̀ la mémoire duquel elle est dédiée.

III. Moignons ćlỵtraux notablement développés, en général bien plus grands chacun que la partie apparente du mesonotum. lícusson distinctement séparé de celui-ci par une suture. ㅇ. - Dernicr segment ventral apparent prolongédans son milieu en une forte pointe obtuse. $\sigma^{x}$. 6. x. aeichi Jaer. du V. - L. mauritanica Oliv. Ent. Ir. 28. 11. 1. Fìg. 5. b. c. $\sigma^{x} \cdot+(1790),-$ Motscl. Etud. Ent. IIr. 16. ơ.? . (Lampronetes) (185i).

Mále. L. 12-16. mil. D’un jame testacé. Pronotum avec une tache discoïdale rose, plus ou moins marruée et parfois légèrement accompagnée de brunàtre; élytres d'un brun plus ou moins clair et parfois un peu jaunâtre, arec une fine marge latérale et suturale testacée; jambes et tarses ordinairement plus ou moins légèrement teintés de brunattre. Pronotum un peu on guère moins long que 
large, point sensiblement rétréci en avant, avec ses côtés droits ou à peu près en arrière. Dernier segment dorsal de. l'abdomen légèrement convexe Jongitudinalement dans son milieu; plus ou moins dilaté-arrondi sur les côtés, puis brusquement mais brièvement rétréci au sommet, avec la partie rétrécie (ou lobe médian) large et notablement arrondie au sominet (fig. 6). . rreeaux précédents prolongés àleurs angles postérieurs externes en forme de dents aiguës, en général assez saillantes. Dernier segment ventral apparent notablement sinué de chaque côté, à pointe médiane brusque et assez étroite. - Femelle. L. 26-35 mil. D'un testacé fauve, avec les trois derniers segments abdominaux d'un jaune testacé pâle; le disque du pronotum, les méso et métathorax, la poitrine et les côtés inféricurs des segments abdominaux (sauf pour les trois dernicrs) largement variés de rose, ceux-ci offrant en outre toutes leurs marges postérieures et latérales de la mème coulcur. L̇cusson notablement rétreci postéricurement. Iluitième segment ventral offrant une petite échancrure ou incision médiane. - Pyrénées-Oricntales, Espagne, Algérie.

Nota. Très certainement celte espèce est la L. mauritanica de M. Motsclılsky et aussi d'Olivior. Cela ressort évidemment des descriptions de ces auteurs et surtout des figures données par Olivier. Je dois cette observation très exacte à mon illustre collègue $\mathrm{M}$. Reiche auquel je me fais un devoir et un plaisir de dédier ce Lampyre. $\Lambda$ joutons qu'Olivier a décrit aussi et figuré comme variété un mâle de la vraie $\mathrm{L}$. mauritanica de Linné ainsi que je l’ai dit plus haut.

Observation. La L. Libani Lap., de Syrie, dont j'ai vu 
deux types dans la collection de M. Chevrolat est très voisine de notre Reichii, mais elle en differe principalement, quant aux mâles, par sa forme bien plus large, par ses élytres offrant une large marge dilatée, déprimée, testacće, avec leur ligne élevée externe bien plus éloignée par suite du bord latéral, par son pronotum marqué d'une tache discoïdale brune, et peu ou point teinté de rose, enfin par le dernier segment ventral apparent point visiblement sinué de chaque côté mais à pointe presque graducllement formée, notablement plus large et plus obtuse; quant à la femelle, par ses moignons élytraux plus longs et plus atténués en pointe en arrière, son écusson plus élevé et plus large à sa base, son corps peu ou point varié de rose, sauf, mais d'une manière moins intense sur le pronotum. - J'ai vu enfin dans la collection de M. Reiche, sous le nom de L. Caucasica Motsch., un exemplaire mâle d'une espèce également voisine de la nòtre, mais s'en distinguant facilement, par sa taille bjen plus petite et la forme toute différente du dernier segnent dorsal de l'abdomen rappelant tout à fait celui de la Zenkeri:

\section{Genre Lampnomza Motsch.}

Mandibules grèles, saillantes, fortement courbées, très ítroites, en pointe simple, munies intérieurement à leur base d'une fine membrane cilice. Pronotum offrant en avant deux grandes taches translueides, très tranchées chez les mâles, plus petites et moins tranchées chez les femelles. - Mäle. Ablomen n'offrant point de petit ar- 
ceau supplémentaire visible au sommet. - Femelle. Taille simplement égale en général à celles des mâles. Corps moins allongé que chez les Lampyris. lilytres représentées par de petils moignons on formed écailles bien marquées et point sinuées postérieurement. Ablomen de huit segments dilatés amincis et subtranslucides sur les côtés.

Le nom de ce genre seul appartient à M. Motschulsky rui n'a connu aucun des caractères importants que je mentionne.

1. ж. мunisanti Kow. An, de la Soc. Ent. Fr. 1851. 587. o

Male. - L. 10-11 mill. Allongé, brun en majeure partic. Tète d'un noir brun, assuz fortement excavéc en dessus. I'ronotum presque semicirculaire, un peu plus large que long, lígèrement rétréci en avant, bisinué à la base, marqué longitudinalement dans son milieu d'une carène fine, mais visible dans toute sa longueur; d'un Jrun obscur sur son disque, cette conleur sc prolongeant anguleusement en avant, d'un brun clair dans le reste de son étenduc, arec deux grandes taches pales semilunaires, translucide;, antirieurement. Écusson plus ou moins testacé. Ellytres brunes, avec une très-fine marge Jilćrale testacíe, offrant trois fines lignes élevées longitudinales plus ou moins marquies dont lit médiaue est la plus complete et de beancoup la plus forte. Poitrine en entier d'un jaune testacé. Cuisses de même; jambes et tarses d’un testacé brunâtre plus ou moins clair. Abdomen brun, avec la marge latérale des segments testacéc ainsi que deux petites taches indétermines sur chacun, bien dis- 
tinctes sur les antérieurs, plus petites, plus obscures et moins visibles sur les postérieurs, nulles sur les deux derniers; le segment apical en majeure partie d'un jaune testacé, le pénultième offrant le plus souvent une petite tache médiane jaunâtre en arrière. - Femelle. L. 9-12 mill. Ovale oblongue, d'un jaune testacé; tête, disque du fronotum, écusson et moignons élytraux d'un brun plus ou moins clair. Ceux-ci bien dévcloppés, assez grands, atteignant en général au milieu du premier segment abdominal en courrant le métanotum. Hanches antérieures fortement transverses, un peu coniques seulement en dedans, très notablement écartées des intermédiaires, dont elles sont séparées par un grand triangle mésosternal largement et profondément excavé dans sa partic antéricure. - Facile à distinguer par ses moignons bruns et par l'organisation de la poitrine semblable à celle qui existe chez toutes les femelles des vrais Lampyris. - PyrénéesOrientales.

Variété $\delta^{x}$ - Pronotum en entier d'un jaune testacé, avec le milieu du disque à peine brünàtre.

2. 2. Boicldien Jacq. du V.

Male. - L. 10-11. mill. Allongé, étroit, parallèle, brun en majeure partie. T'íte d'un noir brun, plus profondément excavée encore que chez la L. Mulsanti. Pronotum en ogive courte, visiblement plus large que long, aussi larice que les élytres dans leur plus grande largeur ì sa buse, notablement rétréci en avant, bisinué à la base, marqué longitudinalement dans son milieu d'une carène fine légèrement interrompue à son tiers postérieur sur un cmpatement lisse du disque; coloré 
comme chez l'espèce précédente, mais avec la tache discoïdale arrondie en avant et remplacée sur le] tiers antérieur par une tache triangulaire d'un testacé pâle. Écusson brun. Élytres comme chez la L. Mulsanti, mais un peu plus fortement ponctuées rugueuses, et avec la ligne élevéc longitudinale externe moins marquée, obsolìte. Poitrine d'un brun testacé. Pattes d'un testacé ì peine brunâtre. Abdomen brun, avec une fine marge latérale testacée, sans taches plus claires distinctes sur les segments antérieurs; le dernier segment en majeure partie d'un jaune testacé, le pénultième, et parfois aussi l'antépénultième, avec une tache centralc flave plus ou moins étendue, - Femelle. Inconnuc. - Basses- $\Lambda$ lpes.

Cette espèce se distingue facilement de la L. Mulsanti par les caractères mentionnés. J'insiste seulement sur la présence de la tache antérieure d'ụn testacé pâle qui s'observe en arant sur le pronotum et parait constituer un caractère très constant. La forme est encore plus allongée que chez la L. Mulsanti, et surtout proportionnellcment plus étroite.' Enfin cette espèce est la seule, sur les quatre que je décris te visu, qui próscnte le pronotum aussi large à sa base. J'en ai vu deux cxemplaires dans la collection de M. Reiche et deux autres dans celle de M. Boieldieu, qui a bien voulu m'en donner un, et auquel je l'ai détice comme un témoignage de mon amitié.

3. .. Dolarouzel Jacq, du V.

Male. - L. 10-12. mill. Assez allongé, proportionnellement assez large, brun en majeure partie. Téte brune, assez fortement excavée en dessus. I'ronotum presque senicirculaire, un peu plus large que long, en général lé- 
gèrement rétréci en avant et le plus souvent visiblement arrondi sur les côtés en arrière, fortement bisinué à la base, marg̨ué longitudinalement dans son milieu d'une carc̀ne fine plus ou moins notablement interrompue à son tiers postérieur sur un empatement lisse du disque; d'un brun obscur sur son disque, cette couleur formant une grande tache en général arrondie en avant, et remplaiéc sur le tiers antérieur par une tache triangulaire d'un testacé pâle traversée cependant par une étroite ligne longitudinale brune; d'un brun clair dans le reste de son ćtendue, avec deux grandes taches pâles semilunaires translucides antéricurement. licusson plus ou moins testacé. Élytres brunes, avec une très fine marge latéralle testacée; offrant trois fines lignes élevées longitudinales assez marquées, dont la médiane est la plus complète, mais est à peine plus forte. Poitrine d'un jaune testacé teinté de brunâtre. Pattes de même. Abdomen brun en entier, avec le dernicr arceau ventral teinté de testacé et les deux pénultièmes offrant chacun une tache centrale flave assuz grande, un peu transverse, mais variant quant $\therefore$ son étendue.-Femelle. L. 8-13 mill. Ovale oblongue, d'un jaune testacé en entier y compris les moignons élytraux; tìte en géneral à peine brunâtre; disque du pronotım à peine plus foncé. Moignons élytraux bien développés,assez grands, dépassant en général le milieu du premier segment abdominal ou même atteignant son sommet en courrant largement le mélanotum. Hanches antérieures transversalement obliques, fortement coniques en dedans, rapprochées des intermédiaires dont elles sont séparées au centre par un trïangle mésosternal petit ou médiocre 
et à excavation antérieure en général tout à fait masquée par elles. - Provence, Var.

Variété ơ. - Pronotum en entier d'un juune testacé, avec le milieu du disque à peine plus foncé; abdomen offrant les taches claires basilaires que l'on observe chez. Ia L. Mulsanti.

J'ai dédié cette espèce à MI. Irelarouzée qui l'a répandue dans les collections de P'aris, et auquel l'entomologie francaise est redevable de la découverto d'un bon nombre d'espèces intéressantes.

4. c. Splendidula Lin. System. natur. édit. 12. Ir. 611. o (1767). - Oliv. Ent. ז. 28. P. 1. Fig. 1. b. of (1790). - Fabr. Ent. Syst. I. Ir. 98. ơ (1792). - Panz. Faun. Germ. 41.8. $\sigma^{\top}+\stackrel{+}{+}(1793-1808)$ - Müll. in Illig. Mag. Iv. 190. 우 (1805). - Gyl. Ins. Suec. 1. 319. ox. ㅇ. . (1808). - Küst. Kitf. Eur, 1x. $27 \cdot \delta^{7} \cdot$. . (1817). - L. noctiluca Jatr. Règn. anim. édit. Nasson, 1. 219. ठ. - L. Sencki, Villaret, An. Soc. Ent. Fr. 1833, 352. o (type). L. Antiqua, Brullé, Exp. de Morée. Artic. 14k. ơ . (1832)

Mále. - L. 9.10 mill. Assez allongé, on général proportionnellement un peu moins étroit que chez la L. Mulsanti, brun en majeure partic. 'Tête d'un noir lrun, forment excavée en dessus. Pronotum presque semicirculaire, visiblement plus large que longr , lírèrement mais sensiblement rétréci en avant, fortement bisinué à la base, marqueć longitudinalement dans son milieu d'une carcene fine mais visible dans toute sa longucur, coloré tout ì fait conme chez la L. Mulsanti. Ĺcusson d'un brun testacé. Lilytres brunes en enticr, offranl trois fines li- 
gnes élevées longitudinales assez marquées dont la médiane est la plus complète et aussi la plus forte. Poitrine l'un jaune testacé teinté plus ou moins de brunâtre. Pattes de mème. Abdomen en entier d'un brun plus ou moins obscur, avec le dernier arceau ventral d'un testacé plus ou moins légèrement brunâtre, et les deux pénultièmes offrant chacun une très grande tache transverse, ou sorte de plaque luisante, occupant toute la partie centrale de chaque arceau, et d'une belle couleur flave avec une marge blanchâtre. - Femelle. L. 6-10 inill. Ovaleoblongue, d'un testacé pâle; tête brune; pronotum coloré comme chez le mâle, mais à teintes un peu plus claires et inoins nettes; anteunes et pattes plus ou moins notablement teintées ou variécs de brunâtre; segments abdominaux paraissant parfois bruns à leur base en-dessus, ou marqués de deux taches brunûtres, par suite de la dessiccation. Moignons élytraux médiocres, ne dépassant pas ou à peine le bord postérieur du métanotum dont ils laissent à découvert tout le ticr's médian. Hanches antérieures et intermédiaires comme chez la L. Delarouzei.Facile à distinguer par son pronotum coloré comme chez le mille, quoique plus claircment, et par la lorièveté de ses moignons élytraux qui, n'étant nullement rapprochés par leurs bords internes, laissent largement à découvert tout le milicu du métanotum. - Europe boréale et centrale. France, Autriche, Italie.

Observation. La L. antiqua de Brullé paraitrait n'ètre pas différente de cette espèce. Quoi qu'il en soit, la description de Brullé n'est pas assez jrécise pour qu'il soit utile de la reproduire. 
L'espèce suivante m'est restée inconnue en nature. J'ai traduit sa description de Küster, en disposant les termes comme dans les précédentes.

L. German Kust. Kûf. Eur. I. 17. o (1844).

Maile. - L. 6 1/2 mill. Oblong, brun en majeure partic. T'ète brune, excavée en-dessus. Pronotum un peu plus large que long, marqué d'une fine ligne longitudinale élevée qui se termine sur un empatement médian; brun sur son disque, d’un brun clair sur les côtés, avec deux grandes taches pâles, translucides, antérieurement. Écusson d'un brun clair, testacé au sommet. Élytres brunes, offrant chacune trois carènes longitudinales dont l'interne est moins marquée et le plus souvent interrompue en arrière, la plus externe commençant à l'épaule, les deux extérieures se réunissant en arrière. Poitrine d'un jaune roussître. Pattes de même et un peu grisâtres. Tarses bruns. Abdomen d'un noir brun, avec le dernier segment d'un jaune gris et le pénultième d'un blanc jaunâtre. - Femelle. Incomnue. - Dalmatie.

M. Küster, en décrivant plus tard la L. splendidula (Ix. 27), dit qu'elle s'éloigne de la L. Germari par sa taille plus grande, la présence de taches lumincuses sur deux segments ventraux, et aussi par son pronotum plus long, les lignes des élytres moins fortes (la médiane ètant presque en carène chez la L. Germari) et les côtés plus droits des élytres. 


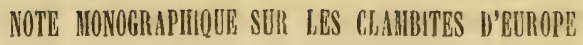

Les très petites mais intéressantes espèces du groupe des Clambites, quoique fort peu nombreuses jusqu'ici, n'ayant jamais été toutes collectivenent décrites in extenso, j'ai cru utile de faire ici ce petit trarail, auquel j'ai été entrainé par l'étude d'une espèce nouvelle pour la faune de France.

Je vais entrer immédiatement en matière, renvoyant à mon Genera (t. I, p. 113) pour les caractères du groupc des Clambites et des deux genres qu'il renferme. Toutefois, je dois auparavant relever une crreur accréditéc jusqu'ici. Les antennes des Clambus sont composées de 10 articles, tout comme celles des Calyptomerus (Comazus), et non de 9 sculement, comme je l'ai cru à tort, ainsi du reste que tous les autres auteurs. Le premier article de ces organes est ípais, subovalaire, le second également épais mais court, le troisième très long et grêle, etc. L'erreur provient non-seulenent de la difliculté de voir en général l'article basilaire, mais encore de ce que les deux premiers articles sontserrés et intimement articulés.

\section{Genre Canumus Fisch. Jacq. du V. Gen. I, 113.}

Corpus globosum rel globoso-ovatum. Caput magnum. Antennic decem articulatx, articulo tertio valde clongato. Pronot:um breve, laterilus anguslatum afque rotun- 
datum. Elytra apice oblusa vel subacuminata. Abdomen segmentis quinque apparentibus infra compositum.

1. c. Amardilo De Geer Mém. Iv. 220 (Dermestes armadillus) (1774). - Gyl. Ins. Suec. I. 190 (Dermestes) (1808).-Fairm. et Lab. Faun. Fr. I. 327 (1853).-Jacq. du V. Gen. I. pl. 38, fig. 188 (18\%i).-Dermestes convexus Marsh. Ent. Brit. 1. 73? (1802). $-\Lambda$ gathidium atomarium Sturm. Deuts. Faun. II. $63(4807)$, -Agatlidium punctulum Beck. Beitr. 8 (1817).

Globoso-ovatus, niger, nitidus, pube grisea suberecta subtilissime sed distincte vestitus; pronoti lateribus anguste rufo testaceo marginatis.

Long. 3/4-1 mill. environ. Corps globoso-ovalaire, fortement déclive en arrière supérieurement, graduellement mais visiblement attenué postérieurement, obtusément subacuminé au sommet. D'un noir plus ou moins intense, luisant, des plus imperceptiblement pointillés, entièrement revêtu d'une très fine pubescence grisâtre dirigée en arrière mais légèrement redressée, peu serrée, très subtile quoique bien distincte à un fort grossissement. Tête très grande, notablement plus large quo longue, largement arrondic antérieurement, anguleuse de chaque côté en arrière, si l'on y comprend les yeux. Bouche d'un testacé roussâtre. Antennes d'un testacé légèrement brunâtre. Pronotum court, fortement transversal, un peu plus large que la tête, aussi large que les élytres à leur base, convexe, fortement déclive sur les côtés, qui sont fortement arrondis, avec ses angles tous arrondis, mais les postérieures plus largement; offrant une bordure latérale étroite et indéterminée d'un roux 
testacé. Élytres largement coupées obliquement de chaque côté à leur base, à épaules formant par suite un angle obtusément ouvert et mousse au sommet. Dessous du corps d'un noir brunåtre. Lames des hanches postérieures offrant à peine une fine marge postéricure roussâtre. Pattes testacées.

Cette petite espèce se distingue parfaitement des suivantes par sa pubescence un peu redressée et bien marquée quoique très subtile. On la trouve dans la majeure partie de l'Europe, parmi les détritus végétaux.

Observation. L'Agathidium atomarium de Sturm ne peut se rapporter qu’à cet insecte. Il est vrai que la figure donnée par Sturm est très mauvaise et que cet auteur représente les antennes et les pattes noires, mais on sait déjà qu'il a fait des pattes et des antennes de Cercyon à son Spharidium irnmundum, lequel cependant, d'après le type, se rapporte au Colenis dentipes.

2. c. Wulsenems Redt. Faun. Lustr. éd. 1. 158 (18i9). - Redt. F. A. éd. 2. 298 (1857). - Fairm. et Lab. Faun. Irauc. I. 327 (1855).

Globoso-subovatus, nigro vel brunnco-piccus, nitidulus, pube grisea depressa brevissima tenuissime dense vestitus; pronoti lateribus late testacco marginatis; elytrisobscure lerrugineis, basi lateribusque infuscatis.

Long. 3/4 mill. environ. Corps globoso-subovalaire, fortement déclive en arrière, graduellement et faiblcment atténué postérieurement, obtus au sommet; d'un noir brun ou d'un brun de poix, tout à fait imperceptiblement pointillé, entièrement revêtu d'une pubescence grisâtre extrêmement subtilo, très courte, complétement 
dépriméc, assez scrrée, donnant ì l'insecte un léger aspect soyeux. Tête comme chez le G. armadilla. Bouche et an1ennes testacées; celles-ci ì massue brunâtre. Pronotum offrant également la forme de celui du C. armadillo, mais un peu moins fortement arrondi sur les còtés et un peu moins largement it ses angles postérieurs; avec une large bordure latérale testacéc bien marquée, mais se fondant peu à peu arec la couleur du disque. Élytres largement coupées obliqquement de charque côté à leur base, à épaules formant par suite un angle obtusément ouvert et mousse an sommet; d'un ferrugineux olsscur, avec leur base et leur bordlatéral bruns. Dessous du corps d'um noir de poix. Lames des hanches postérieures offrant une large bordure postéricure d'un roux testacé. Paltes d'un testacé pâle.

France. Paris. Autriclic. On le trouve dans les vieux fagots et narmi les détritus végétaux. Il se distingue sans peine par sa pubescence tout ì fait déprimée, très courte, mais un peu plus serríe que chez l'Armadillo et cependant moins marquée. Il est en oulre un peu plus petit, un peu plus court et visiblement moins atténué en arrière que ce dernier.

3. c. osinutus Sturm, I)cutsch. Faum. II. 64. (1807) ( $\Lambda$ gathidium). - Gyl. Ins. Succ. 1v. 514 (1827) (Anisotoma). - Faim. ct Lab), Faun. Franc. I. 327 (1833). Clambus armadillus Redt. Faun. Austr.éd. 1. 158 (18:9);

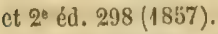

Major, globoso-subhemisphwricus, nigro-brunneus, nitidissimus, supra glaber; pronoti lateribus late ferrugineo marginatis; clytrorum disco late obscure ferrugineo. Variat interdum supra totus ferrugineus. 
Long. 1 a $11 / 4$ mill. Corps fortement globoso-subhémisphérique, très fortement déclive en arrière, afténué tout ì fait au sommet seulement où il est toutefois obtusément acuminé; d'un noir brun très luisant, lisse, glabre supérieurement. Tête comme chez les précédents. Bouche et antennes testacíes ou roussittes; celles-ci à massue plus ou moins brune. Pronotum comme chez le C. armadillo; offrant une large bordure latérale et une étroite bordure basilaire ferrugineuses. Eilytres largement coupées obliquement de chaque côté à la base, à épaules formant par suite un angle très obtusément ouvert et de plus tout ì fait mousse; largement d'un ferrugineux obscur sur leur disque. lessous du corps noir. Lames des lanches postérieures ferrugincuses. Pattes plus ou moins brunes ou roussâtres.

Varictí. Couleur ferrugineuse envahissant tout le dessus du corps. Pattes testacées.

Cette espèce est la plus grande de celles connues de ce genre. Elle a été trouvée en France, en Autriche et en Suède.

Observation. Le C. armadillus de Mr. IRedtenbacher, qui très certainement n'est point lo vrai C. armadillo, me sumble appartenir an G. minutus, d'autant plus que Sturm indique ce dernier comme fprovenant d'Autriche.

4. c. rounctulum fiyl. Ins. Suec. 1v. 515 (Anisotoma) (1827). - Fairm. et Lab. Faun. Franc. 1. 328 (18\%̈).

Minimus, globoso-sublemisphtericus, nigro piceus, nitissidimus, supra glaber; pronoti lateribus anguste rufotestaceo marginatis elytris apice ferrugineis. - Variat 
irterdum brunneus, pronoto lateribus late rufo testaceo, elytrisque ferrugineis basi obscurioribus.

Long. 3/4 mill. ou à peine. Corps globoso-subhémisphérique, déclive en arrière, graduellement et faiblernent atténué postérieurement, obtus au sommet; d'un noir obscur ou un peu brunâtre, très luisant, lisse, glabre supérieurement. Tìte comme chez les précédents. Bouche ferrugineuse. Antennes testacées, à massue un peu brunâtre. Pronotum comme chez le C. armadillo, mais un peu plus étroit sur les côtés, avec ses angles postéricurs aussi fortement et largement arrondis; offrant une étroite bordure latérale d'un roux testacé. Élytres largement coupées obliquement de chaque côté à la base, à épaules formant par suite un angle obtusément ouvert et mousse au sommet; plus ou moins ferrugineuses à leur extrémité. Dessous du corps noir. Lames des hanches postérieures offrant une étroite bordure postérieure d'un roux testacé. Pattes brunes; tarses d'un testacé pâle.

Variété. Tête et pronotum bruns, ce dernier avec une large bordure latérale d'un roux testacé. Élytres d'un ferrugineux devenant brunâtre vers leur base. Dessous du corps d'un brun ferrugineux; lames des hanches offrant une très large bordure d'un roux testacé. Pattes testacées.

Montpellier. Fontainebleau. Suède (Gyl.).

Genre Calyptomenus Redt. Jacq. du V. Gen.I. 11/4.

Corpus ovatum. Caput permagnum. Antennæ decem articulatæ, articulo tertio sequenti modo subæquali. Pronotum brevissimum, lateribus valde angustatum atque subacuminatum. Elytra apice subtruncata. $\mathrm{Ab}$ - 
domen segmentis sex apparentibus , infra 'compositum.

Je regarde comme identiques les genres Calyptomerus Redt. et Comazus Fairm. Cette opinion, émise dans mon Genera, a été adoptée dans les Catalogues de Stettin et de M. de Marseul. Dans le récent Catalogue de M. Schaum au contraire, elle se Irouve rejetée et les deux genres en question sont admis comme propres, en rertu, je pense, d'une assertion de M. Kratz (Berl. Ent. Zeits. 1857, 174) signalant l'espèce de M. Redtenbacher comme différente. 'Toutefois M. Kraatz n'est entré dans aucun détail et s'est borné à diro uniquement que cette dernière est presque deux fois aussi grande que le Comazus enshamensis. La question était cependant assez importante pour mériter une explication. Malgré l'assertion de $\mathbf{M}$. Kraatz, je crois plus que jamais, non seulement les deux genres, mais encore les deux espèces, identiques. En eflet nous trouvons, dans la nouvelle édition de la Fauna Austriaca de M. Redtenbacher, la note précieuse suivante : « Ce Coléoptère (Calyptomerus alpestris) a été envoyé ì M Hampe par M. Mulsant, de Lyon, comme le Cyrtocephalus cephalotes Dejean. „or ce dernier insecte n'est autre que le Clambus (Comazus) enshamenis Steph. (Voir Lacord. Gen. Ir. 223). Donc la question est tranchéc par M. Redtenbacher lui-même, sans qu'il s'en doute toutefois. De plus, lassertion de M. Kratz, touchant la taille plus grande du Calyptomerus alpestris, est réfutée par l'indication (1/3 de ligne) que donne $\mathbf{N}$. Redtenbacher.

1. o. Dubin Marsh. Ent. Brit. I. 234 (Scaphidium) (1802). - Wollast. Cat. Mader. 147 (1857). - Clambus cnshamensis Steph. I11. II. 184 (18:9), - Steph. Man. 
107 (1839).-Comazus enshamensis Fairm. et Lab. Faun. Fr. I. 328 (1850). - Calyptomerus enshamensis Jacc. du V. Gen. I. pl. 38, fig. 189 (1856).-Calyplomerus al-

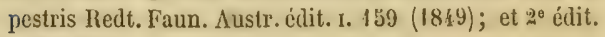
298(1857).

Ovatus, subnitidus, brunneo vel interdum testaceoferrugineus, pube brevi flavescente depressa subtiliter dense vestitus; pronoti lateribus elytrorum que apice didilutioribus.

Long. 1 à 1 //4 mill. Corps ovalairc, globuleusement convexe en avant, fortement déclive en arrière, très visiblement atténué postérieurement mais presque tronqué au sommet; d'un brun ferrugineux ou d'un ferrugineux testacé, avec les côtés du pronotum et le sommet des élytres plus clairs, celles-ci en général au contraire plus obscures a leur base; légèrement ou peu luisant, imperceptiblement pointillé, entièrenent revêtu d'une fine pubescence jaunittre, courte, déprimée, mais assez serrée. Tète énorme, aussi grande que le prothorax et presque aussi large, arrondic antérieurement, subarrondie sur les côtés en arrière. Bouche et antennes testacées. Pronotum extrèmement court, ì angles postérieurs entièrement nuls, le bord postérieur ćtant confondu avec les latéraux de manière à former un grand arc non interrompu jusqu'aux angles antérieurs qui sont saillants latéralement et suljacuminés. Élytres plus largement mais moins obliquement coupées de chaque côté à la base que chez les Clambus. Pattes d'un testacé pâle.

France. Paris. Angleterre. Autriche (Redt.). Madère (Wollast.). 


\section{NOTE SUI LE GENRE XANTHOSPIIERA FAIRA.}

ET DEScription D'UNE ESPÈce NOUVELlE DE COLE.yis.

Tout récemment M. Fairmaire (Annal. de Ia Soc. ent. de France, 18.59, 29) a cru tevoir créer un genre nouveau, Xanthosphara, pour une espèce nouvelle du groupe des $\Lambda$ nisotomites. Ce genre, dit l'auteur : " est très voisin des Triarthron, dont il se dıstingue facilement par les tar. ses postérieurs de quatre articles; et il en présente tout le ficiès; quant au genre Colenis son mésosternum est caréné et la massue de ses antennes est réellement de cinq articles et non de trois, comme le dit Erichson, le septième article étant plus gros que les précédents et que le suivant; il a en outre les pattes et les antennes plus grêles, et la sculpture des élytres est toute différente. ”

Supposons le genre Xanthosphera parfaitement distinct, M. Faimaire a tort de le rapprocher des Triarthron car la structure des tarses prime, comme chacun le sait, les autres caractères chez les Anisotomites. Cet auteur de plus attache une trop grande importance it la structure de l'antenne de la Colenis dentipes. Ein eflet, le septième article est trop petit, equoiqu'mn peu plus grand que les contigus, pour permettre de considérer la massue comme de cinc articles. Beaucoup d'autres insectes sont dans le cas de la Colenis dentipes, et, tont proche, dans le genre Agathidium nous trouvons, suivant les espices, le huitième article de l'antenne igal au septieme on un peu plus grand. Quant au mesositrnum, caréne chez la Cole- 
nis dentipes et simple chez la Xanthosphæra, il ne constitue pas un caractère assez important pour autoriser la formation d'un genre, ct, du reste, dans la même famille, nous le retrouvons aussi caréné ou simple dans le genre Chovela Latr. (Catops Payk.). M. Fairmaire a donc eu tort d'établir le genre Xanthosphara, car cette coupe n'est point suffisamment distincte du genre Colenis.

L'espèce nouvelle que je vais décrire nous en fournit une preuve formelle par les caractères qu'elle présento. En effet, elle offre la massue des antennes de trois articles, dans le sens que M. Fairmaire l'entend, comme sa Xanthospliæra, mais elle a ces organes plus longs que ceux de la Colenis dentipes à l'inverse de celle-ci; elle présente le mésosternum caréné il est vrai, mais moins fortement, en avant surtout où la carène devient obsolète, que chez la Colenis dentipes; enfin elle offre lia sculpture des élytres analogue à celle de ce dernier insecte.

L.e genre Colenis renfermera donc aujourd'hui trois espèces européennes qui peuvent se grouper ainsi qu'il suit : I. Mésosternum simple. Septième article des antennes égal aux contigus. C. Barnevillii Fairm. - II. Mésosternum caréné. - $\Lambda$. Septième article des antennes égal aux contigus. C. Bonnairii J. du V. - B. Septième article des antennes un peu plus grand que les contigus. C. dentipes Gyl.

colenin monnatril Jacq. du V. Ovata, convexa, flavo testacea, sat nitida; pronoto minus brevi, subtilissime punctulato; clytris subtiliter punctato-striatis, interstitis confertim subtiliter transversim strigosis. Mas. Femoribus posticis compressis, sat longe acuteque unispinosis. 
Long. 2 mill. - Corps ovale, convexe, assez luisant, en entier d'un jaunc testacc. Antennes un peu plus courte que la tête et le pronotum, à deuxième article plus épais que le troísième qui est an moins moitié plus long que lui; le septième égal aux contigus; le dernier article de la massue un peu plus étroit que les deux précédents. Tète finement pointilléc. Pronotum environ deux fois aussi large que long, visilblement moins court que chez la C. dentipes, ì peine rétréci en avant, légèrement arrondi sur les côtés, visiblement sinué de cliaque cûté à son bord antéricur et légèrement aussi à sa base, avec ses angles postérieurs obtus et un peu arrondis même au sommet ; convexe sur son disque, très subtilement pointillé. Élytres légèrement mais distinctement striées, ì stries finement ponctuces, neu visibles an sommet, la suturale toutefois au contraire fortement enfoncée en sillon en arrière. - Meile. Cuisses postírieures larges, fortement comprimées, avec leur hord postéricur formant simplement un angle a peu près droit et peu saillant en dehors au sommet, mais notablement dilaté-arrondi presque dans toute sa mortié basilaire et muni sur cefte dilatation d'une forte épine aiguë assez longue et assez élroite. - Femelle inconnue.

La G. Bonnairii offre un faciès intermédiaire entre celui de la Colenis dentipes et des A garicophragus, ou mène plutôt plus voisin du faciès de ees derniers. Elle a été découverte dans la forêt de Fontianebleau par M. Bonnaire qui m’a généreusement cédé, par l'intermédiaire de I. de Bonvouloir, le seul exemplaire qu'il eùt pris. 


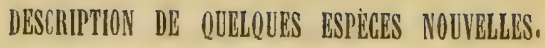

\section{1 adelops soswouloirii Jacq. duV.}

Subovatus, fore depressus, rufo, vel interdum flavo, testaceus, subtiliter fulvo pubesce:n; ; octaro anteunarum articulo nono tantum paulo angustiore; thorace magno, lato, crebre subtilissime punctulato; elytris postice valde attenuatis, subtilissime transwersim strigosis, dor:0 stria juxta suturali obsoleta notatis. - Mlas tarsis anticis quinque articulatis, cum articulo primo valde dilatato, maximo, secundo tertionue minutis sed etiam dilatatis, insignitus.

Long. $32 / 3-4$ 1/2 mill. Corps subovalaire, large en avant, notablement atténué en arrière, très peu convexe ou presque déprimé supérieurement, en entiel d'un roux testacé, ou parfois d'un jaune testacé plus ou moins clair, assez deusément revêtu d'une très-fine et courte pubescence déprimée jaunûtre. Antennes très allongées, très grtèles, un peu plus longues que la moitié du corps, à peine épaissies vers l'extrémité; leur's six premiers articles beancoup plus longs que larges, cylindri(ques, subégaux, le $7^{\circ}$ de même mais faiblement ćpaissi au sommet, le $8^{\circ}$ plus court et un peu plus étroit, le $9^{e}$ subígal en longueur au $8^{\circ}$ mais un peu plus épais, le $10^{\mathrm{e}}$ un peu plus court que le précédent, olsconique, le dernier ovale oblonrs, acuminé. Této verticale, trè:finement pointillée, luisante. Pronotum grand, faiblement convexe, un peu luisant, en 
géníral légèrement plus lirge, que les élytres à leur base principalement chez les mûles, moitjé plus large que long, légèrement arrondi sur les côtés en arrière, rétréci notablement en avant dans sa moitié antérieure, échancré antérieurement, lisinué à la base, avec ses angles postéricurs aigus, un peu saillants en arrière et recouvrant les épaules; densément et très finement pointillé. Élytres presque déprimées sur leur dos en avant, simplement arquées légèrement sur les côtés, fortement atténuées en arrière et arrondies clracune au bout; densément et finement striées transversalement, avec une strie juxtasuturale très légère vers sa base ou vers son milieu, mulle dans le reste de son étendue, parfois même en cntier tout à fait obsolète. - Mâle. Tarses antérieurs de cinq articles distincts; a vec le premier fortement dilaté, en carré long, aussi grand que les trois suivauts rúunis, le second bien plus petit maís dilaté aussi, brièrement cordiforme, le troisième pilus petit encore mais toujours un peu dilaté, tous trois munis de Jrosses de poils inférieurement.

Cette magnifique espèce, la plus grande de toutes celles connues du mème genre, relppelle un peu par son faciès la Choleva depressa Murray. Je l'ai décourerte dans diverses grottes des l’jrénces-Orientales, et je l'ai dédice ì mon ami M. II. de Bonvouloir comme un tímoignage de ma sincère affection.

2 machaerlles arariae Jacq. duV.

Oblongus, clongatus, rufo teslaceus, parce pubescens; antemnis, palpis, pedibusque testaccis ; capite elongatoabsque oculis; thorace suborato, lateribus antice rofun, 
dato-dilatato, basin versus attenuato, Ixvi; elytris sat fortiter punctatis.

Long. 1 2/3 mill. Allongé, oblong, assez convexc; d'un roux testacé, assez luisant, revêtu d'une très tine pubescence jaunâtre peu serréc. Tête deux fois au moins aussi lorıgue que large, un peu dilatée et subangulée de chaque còté en arrière à la place des yeux, formant une sorte de rostre en avant; avec la partic antérieure du front élevée et divisće par unc forte impression médiane en deux tubercules arrondis mais bien marqués et assez écartés; notée sur la partie postéricure de celui-ci de deux petites fossettes; affrant enfin sur le vertex une légère impression médiane ct une fine carène lorigitudinale dans celleci. Palpes maxillaires très allongés, à deruier article grand, long, cultriforme. Antennes insérées sur les tubercules frontaux, atteignant environ ì la base du pronotum, peu épaisses; a $f^{\text {er }}$ article aussi long que les quatre suivants réunis, légèrement épaissi, subcylindrique, $2^{\circ}$ globoso-ovalaire, 3 à 9 étroits, graducllement un peu plus courts et à peine plus épais, $10^{\circ}$ subglobuleux, dernier assez grand, ovale oblong. Pronotum assez convexe, au moins aussi lon que lar:re, notablement dilaté arrondi sur les còlés en avaut, resserré tout ì fait au sommet, graduellement rétréci en arrière, lisse, avec une ligne enfoncée transverse au-dessus de sa base. Elytres assez convexes, fortement bisillonnées chacune à leur base, le sillon interue se prolongeant le long de la suture, assez fortement unais peu densément ponctuées. $\Lambda$ bdomen rebordé latéralement comme chez les l3ythinus. Pattes assez longues et Erìle's sintout les postéricures; cuisses antérieures légère- 
ment épaissies, les postérieures à peine; jambes de cette dernière paire légèrement courbées et sinurés en dedans au sommet; tarses terminés par un seul crochet.

Le genre Machærites, fondé récemment sur un petit Psélaphide (MI. spelæus) découvert dans une grotte de la Carniole, doit prendre place avant les Bythinus. Je le décrirai dans le supplément de mon Genera. L'espèce nouvelle que je publie est des plus intéressantes pour la Faune de France. J'en ai découvert un seul exemplaire dans une grotte des Pyrénées-Orientales. J'ai dédié ce rare et curieux insecte à la compagne dérouée qui, partageant souvent mes fatigues, récolta avec moi ce remarquable Psélaphide nouveau, l'Adelops Bonvouloirii, la Cardiomera Genei, l'Ebaeus Alicianus et beaucoup d'autres richesses entomologiques.

3. x.estevn muscornm Jacq. du V. Aptera, nigra, longius pubescens, fortius minus crebre punctata; ore antennis, pedibusque rufo-testaceis; elytris thorace vis duplo longioribus, fortius punctatis.

Long. 3 1/3 - $41 / 3$ mill. Forme voisine de celle de la L. punctata. Corps aptère, noir, assez luisant, fincment revêtu d'une pubescence grisâtre visiblement plus marquée, plus longue et plus redressée que chez le L. punctata. Bouche et devant de l'épistôme d'un roux testacé. Antennes de même, parfois légèrement rembrunies dans leur milieu, visiblement plus longues que celles de la L. punctata, mais de même épaisseur. Tête comme chez la L. bicolor, mais plus fortement ponctuée. Pronotum comme chez la L. punctata, mais un peu plus brusquement et fortement rétréci en arrière de la dilatation 
des côtes, un peu plus fortement ponctué encore, mais toutefois moins densément d'une manière très sensible. Élytres à peine le double plus longues que le pronotum, un peu plus fortement mais à peine moins densément ponctuées que chez la L. punctata. Abdomen finement mais assez densément ponctué. Pattes d'un roux testacé.

Cette espèce voisine de la $\mathbf{L}$. punctata s'en distingue facilement par sa coloration, sa ponctuation encore plus forte mais moins dense, la pubescence plus marquée et plus longue, etc. Ces caractères de plus empêchent de la confondre avec aucune autre. Je l'ai trouvée dans les Pyrénćes-Orientales, sous la mousse bumide, au bord des fontaines. - Parfois la tête et les élytres deviennent un peu ferrugineuses.

4. Ebacus alicianus. Jacq, du V. Niger, parum nitidus, antennis basi late quatuorque pedibus anticis testaceis; thorace, maris albido, feminæ rufo-testaceo, vitta lata longitudinali media nigra antice fortiter bifida notato; capite, clytrorumque apice processu cyathiformi brunneo aucto, maris, albidis.

Long. 2 1/4 mill. Corps noir, peu luisant, finement revêtu d'une courte pubescence blanchàtre extrêmement subtile. Antennes testacées, plus ou moins rembrunies vers le sommet, un peu plus épaisses chez le mâle. Tête en entier d'un blanc flave, sauf les yeux, chez le mâle, avec le front largement mais légèrement excavé; noire et à peine très faiblement impressionnée chez la femelle. Pronotum moitié plus large que long, un peu plus large chez les mâles, fortement arrondi sur les cotés et à ses angles postéricurs, moins à ses angles antérieurs, légè- 
rement convexe, lisse, d'un blanc flave chez le måle, d'un testacé rougeâtre cliez la femelle, offrant longitudinalement dans son nilicu, chez les deux sexes, une large bande noire diviséc en deux branches étroites dans sa moitié antérieure par une très profonde incision. Blytres densément mais imperceptiblement pointillées, unicolores et simples chez les femelles, avec une tache d'un blanc flave commune au milieu du sommet chez les mâles, dans lesquels chaque élytre présente à ce dernier un petit appendice irrégulièrement cupuliforme. Les quatre pattes antérieures testacées, avec les cuisses brunâtres it la base chez les femelles; les pattes postérieures brtmes avec une large ligue longitudinale sur la face des cuisses, la base et le sommet des jambes testacés notamment chez les máles.

J'ai pris deux exemplaires de cette jolie pelite espèce, voisine de l'Ebacus albifrons, dans les Pyrínées-Orientales. Puisse le nom de cet Ebaeus, charmant comme elle, rappeler longtemps à ma chère Alice les joies enfantines que lui ont sourent données les insectes.

\section{Aspidiphorus Lareynu Jacף. du V.}

Subhemisphæricus, subglobosus, niger, nitidus; antennis, cum clavâ obscuriore, pedibusque rufis. Capite pronotoque subtiliter sat crebre punctatis, pube griseâ brevi vestitis, elytris punctato-striatis, interstitiis sparsim subtiliter punctulatis, pube griseî subseriatim dispositâ vestitis.

Long. $13 / 4$ mill. Corps subhémisphérique, très fortement convexe, d'un noir assez luisant. Antennes d'un rouge ferrugineux, avec la massue assez obscure. Tête 
finement pointillée, revètue d'une courte pubescence grisâtre. Pronotum court, transverse, près de trois fois aussi large que long, notablement défléchi sur les côtés où il est très finement rebordé, très fortement bisinué, largement prolongé vers l'écusson dans son milieu en un lobe tronqué au sommet; finement et assez densément ponctué, revêtu d'une courte pubescence grisâtre. Écusson arrondi, finement pointillé. Élytres courtes, larges, légèrement arrondies sur les côtés et fortement en arrière; distinctement mais peu profondément ponctuées striées; à intervalles plans, offrant de très petits points épars; revêtues d'une pubescence hérissée assez courte, paraissant dans son ensemble former une série longitudinale sur chaque intervalle. Pattes d'un rouge ferrugineux.

Cette espèce se distingue de l'Aspidiphorus orbiculatus, seule jusqu'ici connue de ce genre, par sa taille notable. ment plus grande, son pronotum plus fortement sinué et prolongé en arrière à la base, la pubescence de ses élytres plus distincte et paraissant, comme je l'ai dit, former des séries longitudinales. Elle a été découverte dans la Dordogne par mon malheureux ami Philippe Lareynie, à la mémoire duquel je l'ai dédiée.

\section{Apate xyloperthoirden Jacq. du V.}

Elongata, cylindrica, nigra, nitidula ; antennis tarsisque rufo-testaceis; pronoto cucullato, latitudine paululum longiore, apice emarginato, dimidia parte anticá tuberculis acutis retrorsis tecto, dimidiâ parte posticâ lateribusque subtiliter punctato; elytris parallelis, postice retusis atque leviter bisulcatis, supra subrugulosis, antice sat cre- 
bre fortius, postice sensim obsoletius, punctatis, pube brevi aureâ parce vestitis.

Long. 1/2 mill. Corps allongé, cylindrique, d'un noir assez luisant. Tête finement ponctuée-rugucuse. Antennes en entier d'un testacé rougeatre, avec leurs deux premiers articles plus courts que le funicule; à massue subégale en longueur à ce dernier, ovale oblongue, assez lâche, subcomprimée, et à articles légèrement et obtusément prolongés en dedans. Pronotum cucullé antérieurement, un peu plus long que large, faiblement ou à peine arrondi sur les côtés, échancré au sommet, fortement déclive en avant, avec ses angles postérieurs arrondis; revêtu au moins dans sa moitié antérieure de petits tubercules aigus dirigés en arrière, assez serrés, plus forts en avant sur les côtés, graduellement moins marqués en arrière; finement ponctué dans sa moitié postérieure; revêtus de très petits poils dorés assez épars. Écusson très petit, en forme de point élevé. Elytres parallèles, cylindriques, à épaules assez saillantes, postérieurement rétuses, et largement sillonnées de chaque côté dans l'impression, arrondies au sommet, légèrement rugueuses supérieurement et de plus recouvertes de points assez serrés, plus forts à la base, graduellement plus petits en arrière et obsolètes au sommet; un peu brunâtres postérieurement; revêtues de très petits poils dorés, courts et peu serrés. Dessous du corps noir. Pattes d'un brun noir, avec les tarses d'un testacé ferrugineux.

Je dois cette espèce à l'obligeance de M. Guérin Méneville. Elle provient des environs de Nice où elle parait, dit-on, vivre dans les joncs. Elle ressemble extremement 
au premier abord à la Xylopertha sinuata, et peut-être on la confond parfois avec elle, mais elle appartient très certainement au même genre que les Apate capucina, varia, etc.

\section{DIAGNOSES}

\section{DR DEUX GENRBS MOUPEAUX DO GHOUPR DES DASYTTTES}

Comme le travail que je viens de terminer presque on entier, pour mon Genera, sur le groupe des Dasytites, ne paraîtra qu'à une époque encore assez éloignće, je reproduis ici les principaux caractères de deux genres nouveaux que j’ai cru devoir créer dans le groupe en question, afin de ne pas être exposé à perdre le fruit de mes recherches.

Genre xobonyx Jacq. du Val. - Labrum transversosubquadratum. Mandibulæ vix apice bidentatæ. Maxillæ lobis brevibus, externo fere quadratim latitudine paulo longiore. Palpi maxillares articulo ultimo late oblique truncato. Labium brevo; cum ligula fortiter profunde emarginala. Antennæ filiformes. Tarsi articulo primo ultimo subxquali ; unguiculo utroque intus lobo membranaceo connato, apice modo libero, longitudineque : fere sequali, aucto - Mas valde insignis tarsorum anti"i., còrum articulo primo brevissimo, secundo tertioque obli-

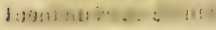


que cæsis, intus apice paulo productis, margine interno leviter dilatatis atque subtiliter breviterque dense pectinatis.-Type: Dasytes aneus F. Gyl. (D. ciliatus Grael.)

Genre ceralus Jacq du V. - Labrum transversum. Palpi maxillares articulo ultimo oblongo-subcylindrico, apice truncato. Antenna breves, articulis decem distinctis modo compositie, apicem versus sensim incrascatic intusque obtuse serratic. Tibia compressæ, apicem versus dilatato extusque parce spinosa. Tarsi sat validi, subtus spinosuli ; articulo primo secundo valde breviore desuperque agre distinguendo. Unguiculi basi intus fortiter dentati. - Type : Dasytes rubidus Schoenh.

Ces deux genres sont très distincts de toutes les autres coupes génériques du groupe des Dasytites.

\section{NOTE SUR LE GENRE ANTIDIPNIS WOLL.}

ET UNE COUPE NOUVELLE VOISINE HOYOEODITNIS JACQ. DU TAL.

Sans contredit la critique, des faits surtout, rend d'immenses services à la science en contrôlant et confirmant les observations exactes, éclairant les questions controversées et détruisant peu à peu l'erreur. Toutefois pour rendre de tels services il faut que la critique, lorsqu'elle n'est pas d'appréciation, s'appuie sur des preuves certaines. Si elle est basée sur des présomptions, entremélée de doutes ou de suppositions gratuites, elle devient 
un véritable fléau, jetant l'irrésolution dans les esprits, compliquant des questions parfois simples, ou meme remplaçant la vérité par l'erreur. Malheureusement les entomologistes allemands, toujours pressés de corriger leurs voisins à tort ou à raison, se laissent trop souvent entrainer à critiquer sans preuves ni matériaux suffsants, sinon même sans nécessité. Je vais en citer un exemple.

M. Kraatz (Berl. Ent. Zeits. 1859, p. 73) publie une assez longue dissertation dans laquelle il s'efforce de prouver, principalement, que mon Colotes rubripes Perr., sur lequel M. Wollaston vient de fonder le genre Antidipnis, n'est probablement qu'une variété de l'Antidipnis (Charopus) punctatus Er., et que je n'ai point connu les deux sexes de mon Colotes Javeti.

Examinons les assertions de M. Kraatz, après avoir toutefois constaté qu'il ne connait point mon C. Javeti en nature.

Le critique allemand débute ainsi : Le C. rubripes J. du V. offre, en dépit de la couleur et de la différence de forme mises en avant par Du Val, une si grande ressemblance avec l'exemplaire typique (unique) du Charopus punctatus Er. (dont notoirement le Colotes Javeti du V. n'est point différent), que leur identité me semble possible ". M. Kraatz fait tont d'abord une supposition gratuite. Pourquoi mon C. Javeti est-il identique au Ch. punctatus Er? Le critique allemand aurait bien dù nous le dire. J'ai constaté cependant, en décrivant mon espèce, qu'elle était bien distincte du C. nigripennis Kust. Iqui cette fois cst certainement identique au Ch. puncla- 
tus Er.). Parti de cette fausse assertion, M. Kraatz s'évertue ensuite, dans une demi-page, à prouver que le C. rubripes, en dépit, comme il le dit, de mes descriptions, n'est point différent du C. Javeti qu'il ne connait pas, puisque celui-ci est identique au $\mathrm{Ch}$. punctatus qu' $\mathrm{l}^{1}$ connaît (mais qui se trouve malheureusement être tout autre). Remarquons en passant ce raisonnement subtil.

Le critique allemand trouve, de plus, étonnant que les deux sexes de mon C. Javeti soient ailés. Par suite il se pose le problème suivant : « Du Val a pris probablement deux différents degrés de colnration du mâle du C. Javeti pour le mâle et la femelle, ou il existe deux espèces voisines chez l'une desquelles la femelle est ailée, tandis qu'elle est aptère chez l'autre. "Problème bien profond! Ou j'ai pris deux mâles pour les deux sexes, ou j'ai vu les deux sexes comme il faut! Ou il existe une seule espèce, ou bien il en cxiste deux ! M. Kraatz est-il bien certain que la vérité soit dans l'un des deux cas? Parlons sérieusement, que trouve-t-il donc de si étonnant dans une femelle d'Antidipnis ailée. Ne voit-on pas déjà des femelles ailées ct aptères suivant les espèces dans le genre voisin Colotes et aussi dans les genres Troglops et Malachius. M. Kraatz tient tellement à son raisonnement ci-dessus qu'il le répète à la fin du second paragraphe dans ces termes : "Le Colotes Javeti est différent du C. rubripes ct alors le G. Antidipnis a deux espèces; ou cela n'est pas, et alors les vraies femelles du C. Javeti sont aptères. n Ce dilemme naïf n'eût vraiment pas été désavoué par ce bon M. de la Palisse. 
Quoi qu'il en soit, M. Kraatz sachant que la femelle du Ch. punctatus Er., qu'il a vue en nature, puisqu'il la signale comme appartenant au genre Antidipnis, est ap'tère, tandis que j'indiquais la femelle de mon C. Javeti comme ailée, eût dủ se tenir sur ses gardes, d'autant plus que je mentionnais des différences notables de couleur, de forme et de ponctuation. Mon C. Javeti, en effet, est si peu identique à l' $\Lambda$. punctatus, qu'il n'appartient pas même au genre $\Lambda$ ntidipnis. Il ne peut être davantage, par conséquent, identique ì l'Antid. rubripes. Aussi je me suis fort amusé de voir M. Kraałz se battre contre des moulins à vent en djscutant cette dernière assertion, et se tourmenter fort mal à propos dans ses suppositions inutiles touchant la femelle de mon C. Javeti.

Je vais maintenant exposer brièvement le résultat de mes études sur les insectes dont il vient d'être question, le manuscrit correspondant de mon Genera ne devant être publié qu’à une date encore assez éloignée.

Parmi les genres européens du groupe des Malachiites, quatre pour moi se font aujourd'hui remarquer par leurs tarses antéricurs de quatre articles seulement chez les mâles, savoir : Traglops, IIomceodipnis, Gen. nov., Antidipnis et Colotes. Le tableau suivant signale leurs caractères essentiels.

1. Palpes maxillaires semblahles dans les deux sexes; à $3^{\mathrm{e}}$ article toujours notablement plus court que le $2^{\circ}$ et beaucoup plus petit que le dernicr.

A. Dernier article des palpes maxillaires médiocro, subégal en longueur au $2^{\circ}$ ou un peu plus long, subovolaire ou très-obscurément sécuriforme. Tête for- 
tement et largement excavée en dessus chez les måles.

G. Troglors Er. Entom. p. 123 (Type. $T$. Albicans Lin.).

B. Dernier article des palpes maxillaires très grand, aussi long que les deux précédents réunis, mais beaucoup plus épais, fortement renflé, brièvement ovalaire. Tête simple chez les deux sexes.

G. IIomoeonirnis J. du V (Type. H. Javeti J. du V.).

II. Palpes maxillaires très-dissemblables dans les deux sexes; à $3^{\circ}$ article grand, beaucoup plus épais que le $2^{\circ}$ chez les màles, petit comme dans la division I chez les femelles.

A. Pulpes maxillaires robustes chez les mâles, à $3^{\mathbf{e}}$ article subégal au dernier, comprimé, subtriangulaire, dernier grand, comprimé, presque en carté un peu plus long que large, fortement tronqué-arrondi au sommet; subfiliformes chez les femelles, à dernier article médiocre, oblong, atténué et tronqué au bout.

G. Antmipnis Woll. Ann. of nat. Hist. 2e ser.xx, 337. (Type. A. Rubripes Perr.)

B. Palpes maxillaires à $3^{\mathrm{e}}$ article un peu plus petit que le dernier chez les mâles, sub-comprimé, en forme de coin triangulaire à sommet interne, dernicr grand, comprimé, graduellement élargi vers le sommet où il est largement tronqué, très obscurément sécuriforme; à dernier article très grand chez les femelles, comprimé, fortement élargi, largement sécuriforme, presque triangulaire.

G. Colotes Er. Entomogr. p. 129 (Type. C.T'rinotatus Er.). Ces diverses modifications des palpes maxillaires sont 
des plus curieuses et distinguent parfaitement entre eux les quatre genres ci-dessus. Elles ont échappé à Erichson pour le genre Colotes, car les palpes maxillaires qu'il décrit sont uniquement ceux de la femelle. Je figurerai tous ces palpes dans mon Genera.

\section{NOTE SUR LE GENRE MICROPEPLUS LATR.}

Après diverses vicissitudes, la synonymic et la délimitation des espèces du genre Micropeplus paraissent aujourd'hui fixées. Erichson, dans son Genera et species Staphylinorum, décrivait le premier collectivement un certain nombre d'espèces. Plus récemment M. Kraaiz,dans le Naturgeschichte der Insecten Deutsclilands, t. 3, décrivait de nouveau plusieurs Micropeplus. Presque en même temps je décrivais deux espèces nouvelles dans mon Genera, t. 2, et dans le catalogue du mème ouvrage; et je signalais le premier des caractères sexuels et spécifiques nouveaux, tirés de la crête abdominale et de la structure des jambes postérieures, caractères échappés aux deux auteurs précédents. Je croyais en même temps devoir signaler quelques erreurs de M. Kraatz. Cet auteur enfin, après avoir réuni divers exemplaires typiques, vient de publier (Berliner Ent. Zeitschrift, 1859, p. 63) une assez longue note sur les trois espèces en litige, savoir : Staphylinoides, Margaritce et Fulvus. La question me semble décidément tranchée et tout a fait résolue dans cette 
note, et j'accupte parfaitement, avec M. Kraatz, l'établissement de la synonymic ainsi qu'il suit :

1. Staphylinoides Marsh., Erichs., J. du V., Kraatz in Berliner. = Var. Fulvus J. du V.

2. M. Margarite J. du V., Kr. in Berl. $=$ Fulvus Kr. in Naturg.=Var. Fulvus Er.

3. $M$. Longipennis Kraatz in Berl, = Staphylinoides Kraalz in Naturg.

Il suit de là que j'ai à bon droit rapporté le Fulvus de M1. Kraatz à mon Margaritæ, et que j'ai signalé avec raison le Staphylinoides de cet auteur comme différent du Staphylinoides d'Erichson. Il est vrai que j'ai rapporté a tort cette espèce de M. Kraatz à mon Margaritæ, mais je ne pouvais faire autrement, puisqu'elle en était très voisine et nouvelle, ce que je ne pouvais supposer. Quant au M. Fulvus d'Erichson, qui n'est qu'une variété, il était impossible de savoir à quoi s'en tenir sans voir le type, et je devais forcément lui rapporter les exemplaires fauves que j'avais sous les yeux. En outre, ne faisant qu'un catalogue, je n'eus osé réunir une espèce décrite par Erichson, alors que la coulcur fauve très tranchée semblait la bien distinguer.

J'ai cru utile, d'une part, de confirmer la question par' l'examen de mes types que $\mathrm{M}$. Kraatz n'avait pu voir, et de l'autre, d'éclairer sur ce sujet les lecteurs de mon Genera. J'aime toujours, en outre, à rendre lommage ì la vérité. 


\section{NOTE SUR LE GENRE HOMAPTERUS}

DE M. FAIRMAIRE.

M. Fairmaire, (Annal de la Soc. ent. de France, 1856, 537) a publié, sous le nom de Subnudus, une espèce, nouvelle de Curculionide qu'il rapporte à tort au genre Strophosomus. J'ai relevé cette erreur (Annal. Soc. en. Fr. 1837, Bull. 63) et signalé l'insecte en question comme appartenant au genre Metallites. M. Fairmaire, tout en étant forcé d'avouer qu'il s'est trompé (Ann. Soc. ent. Fr. 1857. Bull.), cherche à pallier l'erreur commise en créant un genre nouveau, moyen commode de tourner les questions épineuses, mais qui nous conduirait directement à forger un genre pour cliaque espèce ou peu s'en faut. Aussi M. Fairmaire est-il arrivé, à ma grande surprise, à vouloir diviser en trois le genre Metallites. Suivons ici son raisonnement. Cet auteur part des deux divisions suivantes: A. Corps aptère, le plus souvent ovalaire ou ovale oblong; épaules arrondies, non saillantes. B. Corps ailé, toujours oblong; épaules obtusément angulées et saillantes. Il avoue que les épaules de l'insecte en litige sont un peu saillantes (elles sont visiblement angulées et tout aussi saillantes que chez les Metallites marginatus et surtoutmurinus), mais il ajoute que l'espèce étant aptère elle ne peut rontrer que dans la f $^{\text {ro }}$ section, et considère comme bien plus important que l'autre ce dernier caractère. Idée singulière pour celui qui sait combien au con- 
traire la prísence ou l'absence des ailes est chose pou importante, puisque non-seulement les espèces d'un même genre peuvent à tout moment être indifféremment ailćes ou aptères, mais encore parfois les individus d'une seule et même espèce. Il est évident que parti de cette idée M. Fairmaire devait arriver à créer un genre nouveau, Homapterus, et à vouloir séparer des Metallites les M. ambiguus et murinus, sans remarquer le moins du monde que, d'après son système, ces deux espèces ne devraient pas, comme il le dit, former une coupe nouvelle, mais rentrer dans son propre genre Homapterus. Fort heureusement qu'il ajoute: "C'est assezd"un (genre) pour cette fois ». Pour moi je trouve que c'est de trop, affirme que l'insecte en litige offre les caractères essentiels des Metallites, et crois que l'on ne doit subdiviser les genres que lorsqu'il existe des caractères différentiels saillants. Je le crois ici d'autant plus que beaucoup de genres de Curculionides reposent déjà sur des caractères légers, mais non pas très vagues, comme le dit M. Fairmaire, ce qui est fort différent. Je termine par les couclusions suivantes. $1^{\circ} \mathrm{M}$. Fairmaire s'est trompé, il l'avoue, en inserivant son espèce (Subnudus) parmi les Strophosomus. จ Il a tort de créer pour elle une coupe nouvelle (Homapterus), car ce genre, qui de plus devrait comprendro les Metallites marginatus, murinus, etc., ne se distingue du genre Metallites que par un seul caractère (II. Fairmaire n'en pourra citer aucun autre sérieux savoir : Corps aptère. $3^{\circ}$ La division B des tableaux de mon Genera au lieu de porter : Corps ailé, etc., doit porter et.n'en pas moins subsister : Corps le plus souvent 
ailé. Voilà tout. Ceci prouve tout simplement que la science avance et que les caractères se modifient par une étude plus spéciale de chaque espèce, comme on le remarque tous les jours. Telles sont mos convictions intimes, que chacun juge suivant ses idées.

\section{NOTE SUR LE GENRE GAULOSTROPHUS}

DE M. FAIRMAIRE.

M. Fairmaire (Annal, de la Soc. ent. de Fr. 1859, 55) vient de décrire, sous le nom de Caulostrophus, un genre nouveau de la famille des Curculionides. Examinons quelle est la valeur de cette nouvelle coupe générique, après avoir constaté que j'ai sons les yeux le Caulostrophus Delarouzei de cet auteur. M. Fairmaire s'attache principalement à différencier son genre des Strophosomus et l'en trouve bien distinct. Je suis aussi volontiers de cet avis que s'il eút distingué les Caulostrophus des 0tiorhynchus ou même des Apion. Quant aux Brachyderes dont cet auteur a cru Jevoir dire deux mots à peine c'est une toute autre question. Voici les termes de M. Fairmaire : Le faciès de ces derniers insectes (Brachyderes) est très différent, le scrobe s'élargit vers les yeux., Deux assertions erronées! En effet, le Caulostrophus Delarouzei offre un faciès assez voisin des Brachyderes pubescens et surtout lepidopterus, ot, dans tous les cas, ces deux der- 
niers insectes ont un faciès bien plus différent du Brachyderes lusitanicus que du Caulostrophus Delarouzei. Le scrobe ne s'élargit point vers les yeux; au contraire il s'atténue plus ou moins au bout vers ceux-ci, peu visiblement chez les $\mathrm{B}$. incanus et pubescens, bien distinctement chez le $\mathbf{B}$. lusitanicus comme je l'ai figuré dans mon Genera (Curcul. pl. 6, fig. 27 a). En quoi donc le genre Caulostrophus est-il distinct des Brachyderes ? Essayons de chercher dans la description donnée par M. Fairmaire. En comparant attentivement celle-ci avec celle que j’ai donnée dans mon Genera du genre Brachyderes, je constate les seules différences notables suivantes dans la première: Rostre sillonné longitudinalement et à la base; scrobe assez étroit, profond. Or, le caractère tiré du rostre sillonné en travers à la base n'est point générique d'une part, s'il n'est appuyé par des caractères plus importants, et de l'autre nous le retrouvons, quoique à un moindre degré, chez le B. lusitanicus. Le scrobe varie quant à sa largeur et à sa profondeur. Il est assez large chez les B. incanus, pubescens, etc., plus étroit chez le B. lusitanicus où il est au moins aussi étroit ou plus mème que chez le Caulostrophus Delarouzei ; mal limité et peu profond chez ce même lusitanicus, profond et nettement limité dans toute sa partie antérieure chez le B. pubescens, etc. Ajoutons qu'en examinant la nature je ne constate aucun caractère différentiel réel chez le Caulostrophus Delarouzei, et que je trouve chez lui, tout comme chez les Brachyderes, les jambes antérieures fortement sinuées intérieurement vers l'extrémité, caractère passé sous silence par M. Fairmaire. Comme cet auteur pour- 
rait invoquer peut-être comme un caractère distinctif la petite dent que l'on trouve à l'épaulo, effacée toutefois, de son espèce, disons enfin qu'on remarque également eelle-ci chez le $B$. lusitanicus.

\section{REMARQUES ET SYNONYMIES DIVERSES.}

1. Le genre Duvalius Delarouz., dès son apparition (Annal. de la Soc. ent. de Fr., 1859), s'est trouvétrès justement rejeté (Cat. de M. Shaum, 1859). En effet, les caractères invoçués pour le séparer des Anoplithalmus sont illusoires et purement spécifiques. Comme on pourrait penser, ce genre m'ayant été dédié, que j’ai approuvé sa création, il est de mon droit de dire ici que Mr. Delarouzée l'a publié malgré non avis contraire et mes vives représentations.

2. Je me propose, dans le prochain cahier de mes Glanures, de publier sur les Bembidium d'assez longues notes qui n'ont pu trouver place dans celui-ci. Qu'il me soit permis toutefois de dire que, non-seulement je maintiens toutes mes anciennes réunions non adoptées par M. Schaum, mais encore j'espère prouver, car j'ai rassemblédans ma collection des matériaux convenables pour cela, qu'il faut de plus réunir comme variétés le B. normannum an B. pusillum, le B. femoratum au B. Andreæ et le B. oblongum à mon B. tricolor. Ce dernier, n'étant point le vrai tricolor de Fabricius, j'avouerai toujours la 
vérité, doit prendre le nom de B. ripicola $\mathbf{L}$. Duf. et non de B. scapulare Dej., comme le dit M. Schaum.

3. La note suivante, destinée au Bulletin des $\Lambda \mathrm{n}$ nales de la Société entomologique de France et restée inédite par suite de ma démission, mérite d'être connue. * M. Jacquelin du Val (séance du 24. juin 1857) demande a M. Fairmaire s'il connait l'ouvrage dans lequel Sperk a décrit quelques Staphylinides, et sur la réponse négative de notre collègue, il lui demande pourquoi dans sa Faune française on trouve, à propos du Leptacinus ampliventris J. du V., l'observation suivante : ? Peut» être faut-il le rapporter au Xantholinus breviventer " Sperk ». M. Fairmaire répond qu'il n’a eu d'autre raison de dire cela que l'analogie des deux noms.

4. M. Fairmaire (An.S. Ent. de Fr., 1859, 37), après avoir corrigé son nom d'Oxypoda planipennis en 0 . platyptera, parce qu'il existait déjà une 0 . planipennis Thoms, décrit tout à côté (p. 38) une Oxypoda sous le nom de Riparia, sans remarquer qu’il existe aussi déjà précisément une 0. riparia Thoms.

5. M. Kraatz (Berl. Ent. Zeits., 1858, 61) dit que le Staphylinus lavicollis Brul. lui est resté inconnu, et rapporte cet insecte au genre Philonthus. Il est facile, ce me semble, en lisant avec soin la description de Brullé, de se convaincre que c'est celle d'un Quedius, et même, je crois, celle du Q. frontalis Nordm., qui se trouve en Grèce, en effet, d'après Erichson.

6. M. Kiesenwetter (Berl. Ent. Zcits., 1859, 17) pense que le Melanotus subvestitus Brul. est probablement identique au M. crassicollis Er.; toutefois, il l'inscrit comme 
espèce propre dans le Catalogue de M. Schaum. La description de Brullé me semble se rapporter assez bien au M. brunnipes Germ., qui se trouve en Grèce, d'après M. Kiesenwetter lui-même.

7. Le genre Allotarsus Grael (Mém. 1850) adopté et éloigné notablement des Henicopus dans le Catalogue de M. Schaum, est synonyme de ce dernier genre et tout simplement basé sur une des espèces d'Henicopus à articles des tarses simples dans les deux sexes.

8. Le Dasytes rufitarsis Luc,, incrit par M. Kiesenwetter, dans le Catalogue de M. Schaum, dans le genre Dasytes, et sur la place duquel cet auteur dit ensuite (Berl. E. Zeits., 1859, 185) ne pouvoir se fixer, me semble appartenir au genre Dasytiscus de l'auteur allemand et mème être une espèce très voisine de son Dasyt. graminicola. - 9. Le genre Phloiophilus Steph., relégué, dans le Catalogue de M. Schaum, parmi les genres de place incertaine, appartient bien certainement, comme je l'exposerai dans mon Genera, au groupe des Dasytites.

10. M. Mulsant (Opusc. ent. virr, 27) décrit, sous le nom de Velutinus, une variété de Bruchus, qu'i rapporte avec raison au B. rufimanus Sch. C'est un tort, à mon avis, de donner des noms aux variétés, mais, de plus, celle que M. Mulsant signale comme nouvelle, a été dérite et bien figurée depuis longtemps, comme espèce propre, par Herrich Schaeffer (Panz. Faun. Germ., 172, 4).

11. L'Aromia ambrosiaca de M. Muls. (rosarum Luc.), considérée jusqu'ici comme une espèce distincte de l'A. moschata, n'est bien certainement qu'une variété de 
cette dernière. Je soupçonnais cela depuis longtemps, car j'avais reçu de mon père deux ou trois types de l'A. moschata avec un certain nombre d'exemplaires de l'A. ambrosiaca Muls., pris tous dans la vallée de Prades, dont celle de Villefranche, d'où provenaient les types de M. Mulsant, n'est que la continuation. Cette année, j'ai été à même d'observer les faits suivants de visu. L'A. moschata type se trouve sur les saules, dans la vallée de Prades, pêle-mêle avec l'A. ambrosiaca de M. Mulsant. N'ayant trouvé, contre une vingtaine de Moschata, que quatre Ambrosiaca, j'en conclus, d'après l'envoi précédemment mentionné, que les exemplaires à taches rouges sur le pronotum (Ambrosiaca) sont plus nombreux ou moins nombreux que le type, suivant les années et probablement les circonstances climatériques. Sur les quatre exemplaires à taches, deux offraient celles-ci grandes et bien marquées, un les présentait plus petites, chez le quatrième ces taches étaient à peine visibles. On observe, parmi les exemplaires à taches, les mêmes variétés de couleur que chez le type, sauf la couleur noiråtre. Enfin, je n'ai pu trouver aucune différence marquée et constante entre les exemplaires des environs de Paris et ceux des Pyrénées-Orientales. 
Feronia grata Chaud. $(1859)=\mathrm{F}$. cupripennis Fairm. Bembidium excellens Rosenh. (1856)=B. flavo posticatum J. du V. (1855). M. Schaum (Catal.) n’a donc pas le droit de faire prévaloir le nom de M. Rosenhauer.

Aleochara Grenieri Fairm. (1859) = A. crassicornis Lacord. (1835) ơ + .

Georyssus pimeloides Fairm. $(1859)=\mathrm{G}$. carinatus Rosenh: (1856).

Elaphocera Sardoa Ramb. $(1843)=$ E. obscura Géné (1838). nec Er. Ramb.= M. emarginata Sch. (1817):

Anomala villosa Blanch. $(1851)=\mathrm{A}$. devota Rossi $(1790)$. Muls, d'après le type.

\# Osmanlis Blanch. $(\mathbf{1 8 5 1})=\mathrm{A}$ solida Erichs. (1845).

Microrrhagus alticollis Vil. inéd. Ksw. (1858)= M. unicolor Latr. (1834).

Agriotes rufulus Lacord. $(1835)=A$. pallidulus III. (1807).

Cebrio Benedicti Fairm. (1849) = C. Neapolitanus Costa (1847).

Telephorus apicalis Reiche $(1857)=\mathrm{T}$. assimilis Payk (1798) + (dilatatus Redt.), d'après le type.

Malachius bispinosus Curt. (1827) = M. marginellus F. (1801) $\sigma^{\star}$. nec Spinipennis Germ, in Cat. Schaum.

» ovalis Casteln. (1836) = M. cyanipennis Er. (1840) 우. 
Malachius anticus Casteln. $(1836)=$ Ebaeus albifrons

Fabr. (1801) 우.

Anthocomus fagi Motsch. (1854) = A. amictus Erichs. (1840).

Colotes suturalis Motsch. $(\mathbf{1 8 5 4})=\mathrm{C}$. trinotatus Er. (1840). Var $0^{x}$.

Henicopus amphicoma Grael. $($ 1858) $=$ H. scutellaris F.

(1792). Ksw. (1859). D'après des types.

Ptinus agricultor Rosenh. (1856)=P. abbreviatus Boiedd. (1854-56). Certe.

* formosus Rosenh. (1856) = P. Aubei Boield. (1854 et 1856).

D) Solitarius Rosenh. (1856)=P. obesus Luc. (1846). Boield. (185̈6).

Ptinus Duvalii Lareyn. (1853): Boield. (1856) =P. Variegatus Ros. (1792). Var. d'après le type.

Anobium morio Villa $(1835) .=\mathrm{A}$. fulvicorne Sturm (4837).

v rufipenne Duft (1825). =A. fulvicorne Sturm (1837). Var.

Dorcatoma striato-punctatum Cast. (1840)= = Aspidophorus orbiculatus Gyl. (1808).

Cis alpinus Mellié (1848) = G. bidentulus Rosenh. (1847). Bruchus oblongus Rosenh. (1856) $=\mathrm{B}$. tristiculus Sch. (1839). Certe. D'après plusieurs exemplaires provenant du Portugal, chez lesquels la couleur des tarses intermédiaires est très variable.

Erirhinus macropus Redt. (1849). = E. ventralis Steph. (1833).

Hylesinus Aubei Perris (1855). =H. bicolor Brul. (1832). 
Bostrichus alni Muls. (1856).=B. Saxesenii Ratz. (1839). D'après un type.

Purpuricenus Boryi Brul. (1832).=P. KaelheriF. (1801). Muls. Var D. (1839).

- Affinis Brul. (1832). = P. Budensis Goez. Muls.

Cryptocephalus hirtifrons Grael. (1858)=C. Rossii Suffr. (1848).

Timarcha gravis Rosenh. $(1856)=\mathrm{T}$. pimeloides Her. Schæf. (1838).

D rugulosa Rosenh. $(1856)=$ T. maritima Perris (1855).

Luperus sulphuripes Grael. (1858) = Calomicrus foveolatus Rosenh. (18:5).

Argopus rubidus Grael.(1858) = Sphæroderma testaceum Panz. (1795-1808). M. Graells compare son espèce au $\mathbf{S}$. testaceum, mais il a pris le Cardui pour celui-ci. 